\title{
Retraction Note: DNA Barcoding Korean Birds
}

\author{
Hye Sook Yoo, Jae-Yong Eah, Jong Soo Kim ${ }^{3}$, Young-Jun Kim², Mi-Sook Min², Woon Kee Paek ${ }^{1}$, \\ Hang Lee ${ }^{2}$, and Chang-Bae Kim*
}

Retraction Note: Mol. Cells 22 (2006) 323-327

Members of the editorial board have unanimously agreed to retract the article [Mol. Cells 22 (2006) 323-327] for extensively plagiarizing from two other articles published in PLoS Biol. 2 (2004) e312, and in Heredity 97 (2006) 254-255. Molecules and Cells strictly prohibits plagiarism which in the case of scientific publications refers to the act of presenting a study and/or idea as one's own and failing to specify or disclose appropriate sources.

Biological Resource Center/Korean Biolnformation Center, Korea Research Institute of Bioscience and Biotechnology, Daejeon 305-333, Korea, ${ }^{1}$ Natural History Team, National Science Museum, Daejeon 305-705, Korea, ${ }^{2}$ Conservation Genome Resource Bank for Korean Wildlife (CGRB), College of Veterinary Medicine, and BK21 Program for Veterinary Science, Seoul National University, Seoul 151-742, Korea, ${ }^{3} \mathrm{Hankuk}$ Academy of Foreign Studies, Yongin 449-854, Korea

${ }^{*}$ Correspondence: evodevo@smu.ac.kr 